\title{
S1 Supplement.
}

The steady state solution where all living pools are extinct:

$$
H_{C}=0, \quad D_{N}=0, \quad D_{C}=0, \quad P_{C}=0, \quad N=\frac{N_{\text {ext }}}{K_{N L}} .
$$

The steady state solution where the herbivore is extinct:

$$
H_{C}=0, D_{N}=\frac{N_{e x t}}{K_{D L}}-\frac{K_{N L} d_{P}}{K_{D L} r_{P}}, D_{C}=D_{N} C N P\left(1+\frac{K_{D L}}{r_{\min }}\right), P_{C}=\frac{D_{C} r_{\min }}{d_{P}}, \quad N=\frac{d_{P}}{r_{P}} .
$$

The steady state solution where all pools coexist:

$$
\begin{gathered}
P_{C}=\frac{d_{H}+r_{2 H}}{e_{1} r_{1 H}}, \quad N=\frac{r_{1 H} H_{C}+d_{P}}{r_{P}}, \\
H_{C}=\frac{e_{1}\left(r_{\min }+k_{D L}\right)\left(r_{P} N_{e x t}-k_{N L} d_{P}\right)-\frac{1}{C N P} \frac{r_{P}}{r_{1 H}} d_{P} k_{D L}\left(d_{H}+r_{2 H}\right)}{e_{1} r_{1 H} k_{N L}\left(r_{\text {min }}+k_{D L}\right)+r_{P} k_{D L}\left(\frac{\left(1-e_{1}\right)}{C N P}\left(d_{H}+r_{2 H}\right)+\frac{e_{1}}{C N H} d_{H}\right)}, \\
D_{N}=\frac{\frac{d_{P}\left(d_{H}+r_{2 H}\right)}{C N P \cdot e_{1} \cdot r_{1 H}}+\left(\frac{\left(1-e_{1}\right)\left(d_{H}+r_{2 H}\right)}{e_{1} \cdot C N P}+\frac{d_{H}}{C N H}\right) H_{C}}{r_{\min }+k_{D L}}, \\
D_{C}=\left(\frac{d_{H}}{e_{1} r_{\min }}+\left(\frac{1}{e_{1}}-1\right) \frac{r_{2 H}}{r_{\min }}\right) H_{C}+\frac{d_{P}\left(d_{H}+r_{2 H}\right)}{e_{1} r_{1 H} r_{\min }}
\end{gathered}
$$

